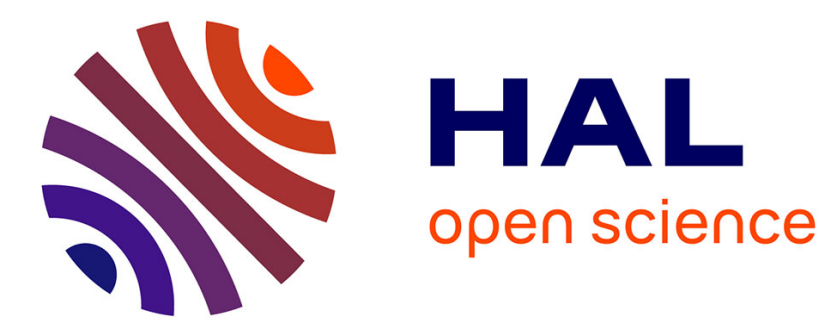

\title{
Properties of type II quadratic solitons excited by imbalanced fundamental waves
}

R.A. Fuerst, Michael Canva, D. Baboiu, George I. Stegeman

\section{To cite this version:}

R.A. Fuerst, Michael Canva, D. Baboiu, George I. Stegeman. Properties of type II quadratic solitons excited by imbalanced fundamental waves. Optics Letters, 1997, 23 (23), pp.1748-1750. hal-00668791

\section{HAL Id: hal-00668791 \\ https://hal-iogs.archives-ouvertes.fr/hal-00668791}

Submitted on 10 Feb 2012

HAL is a multi-disciplinary open access archive for the deposit and dissemination of scientific research documents, whether they are published or not. The documents may come from teaching and research institutions in France or abroad, or from public or private research centers.
L'archive ouverte pluridisciplinaire HAL, est destinée au dépôt et à la diffusion de documents scientifiques de niveau recherche, publiés ou non, émanant des établissements d'enseignement et de recherche français ou étrangers, des laboratoires publics ou privés. 


\title{
Properties of type II quadratic solitons excited by imbalanced fundamental waves
}

\author{
R. A. Fuerst, M. T. G. Canva, D. Baboiu, and G. I. Stegeman \\ Center for Research and Education in Optics and Lasers, University of Central Florida, 4000 Central Florida Boulevard, Orlando, \\ Florida 32816-2700
}

Received August 14, 1997

It was shown experimentally for type II second-harmonic generation in KTP that the family of quadratic spatial solitons exists over a wide range of imbalances for the two fundamental wave inputs. The threshold for soliton formation was measured as a function of both phase mismatch and relative input fraction of the energy in the two fundamental polarizations. Launching unequal fundamental inputs led to an imbalanced three-coupledfield output composition. (c) 1997 Optical Society of America

Quadratic spatial solitons (QSS's) are unique members of the generalized soliton family because they consist of multifrequency waves that self-trap each other by strong energy and phase exchange between the interacting waves. ${ }^{1-3}$ The simplest case occurs for second-harmonic generation (SHG). The existence of both one-dimensional (1D) solitons (which diffract in one transverse dimension) and two-dimensional (2D) solitons (which diffract in two transverse dimensions) was observed experimentally. ${ }^{4-6}$ Related phenomena such as beam steering and soliton collisions were also reported. ${ }^{7,8}$ In the $2 \mathrm{D}$ case KTP with a cut that required type II SHG, corresponding to a three-wave interaction with two input fundamental waves, was used. However, in these experiments the two orthogonal input polarizations for the fundamental beams were of nearly equal amplitude. This geometry was chosen because most of the theoretical literature deals with the simpler case of type I SHG, and for equal fundamental amplitudes the type II geometry becomes degenerate with the type I case yet differs because of the interaction between the two fundamental fields. In the KTP beam steering and switching experiments that involved walk-off between the interacting beams, slightly unequal amplitudes for the fundamentals were used for biasing of the directions into which the resulting soliton propagated. However, it was not clear that there was an extended family of QSS's specific to type II interactions in two dimensions with properties such as their fractional composition that are different from those known for type I interactions. In fact an extended family was discussed theoretically by Peschel et $a l .{ }^{9}$ for the $1 \mathrm{D}$ case and by Buryak et al..$^{10}$ for the 2D case, and solitons with properties different from those in type I geometries were predicted. All-optical switching based on unequal fundamental components with 1D type II solitons was studied numerically. ${ }^{11}$ In this Letter we report experimental studies of the properties of type II 2D QSS's with a variable ratio between the fundamental energies. Specifically we studied, for different phase-matching and excitation fundamental energy imbalance, how the soliton threshold and the equilibrium state (fractions of energy in the three different field components) varied.

0146-9592/97/231748-03\$10.00/0
The experimental system studied was a KTP crystal cut for type II phase matching $\left(\rho_{\omega}=0.19^{\circ}\right) .^{4} \mathrm{~A}$ pulsed (35-ps FWHM) fundamental Gaussian-shaped beam at $1064 \mathrm{~nm}$ was focused to a $20-\mu \mathrm{m}$ (FWHM) spot size at the entrance face of the crystal. The polarization of the laser beam could be varied via a halfwave plate, producing any ratio of the $e$ beam-o-beam fundamental amplitudes that were excited in the crystal. In this arrangement the two fundamental beams started with zero phase difference. The second harmonic was generated with a walk-off angle $\rho_{2 \omega}=0.28^{\circ}$. Furthermore, we could angle tune the crystal to obtain different values of the phase mismatch, $\Delta k L=$ $\left(k_{3}-k_{1}-k_{2}\right) L$. The KTP crystal was $1 \mathrm{~cm}$ long, which corresponds to roughly five diffraction lengths for the input conditions. Energy measurements were done with a $1-\mathrm{cm}^{2}$ pyroelectric energy meter, and we performed spatial analysis by imaging the soliton on a video camera. ${ }^{4,6,12}$

First the pulse threshold energy was measured experimentally as a function of phase mismatch for balanced input conditions, i.e., equal excitation of the two orthogonally polarized fundamental fields. The threshold is defined as the pulse-energy level at which the width and brightness of the generated solitons appear stable despite noise in the peak intensity of the incident laser. ${ }^{6,12}$ Based on previous research, ${ }^{12}$ this approach overestimates by as much as $30 \%$ the energy required for output fundamental pulses equal in width to the input pulses. However, this approach does provide a consistent and reproducible criterion. The results, illustrated in Fig. 1, show that the threshold rises for $\Delta k L<0$ with increasing $|\Delta k L|$ from the phase-matching point. For $\Delta k L>0$, the threshold is constant over the range studied. For positive phase mismatch this behavior appears to disagree with the theoretical results of Torner et al., ${ }^{13}$ in which the threshold rose linearly on both sides of phase matching with increasing $|\Delta k L| .{ }^{13}$ However, in Ref. 13 it was assumed that a stationary QSS is launched at the input, including a second-harmonic beam. The curves that are specific to only fundamental input lie in the continuum above the minimum criteria demonstrated by Torner's existence curves. ${ }^{3,13}$ This observation is 


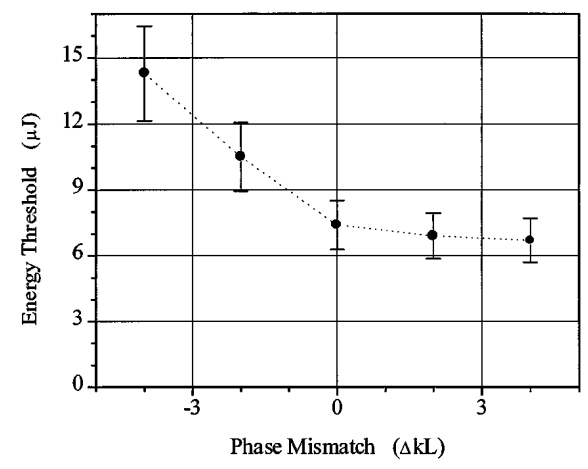

Fig. 1. Soliton-formation threshold energy for equalintensity fundamental inputs polarized along the ordinary and extraordinary axes versus different phase mismatches.

consistent with our previous research in which a second harmonic was launched along with a fundamental seed and the fundamental field of the soliton was generated through propagation. ${ }^{6}$

The pulse-energy threshold was also measured for different values of phase mismatch as a function of different imbalanced fundamental launching conditions. In general the minimum threshold occurs near equal excitation of $o$ and $e$ polarizations, and the threshold rises sharply as the imbalance is increased (Fig. 2). Since the second harmonic is generated from the two fundamentals, one photon from each wave is needed for production of one harmonic photon. As the input polarization is rotated away from the balanced condition, progressively fewer photons would be available at the input in one of the fundamental waves if the total energy remained constant. Actually, by plotting the composition of the $e$ - and $o$-polarized fundamental waves at the input, one can see a flat region formed near $3 \mu \mathrm{J}$ that represents the minimum energy required in the weak component of the fundamental for QSS formation to occur. At some imbalance level there will be insufficient photons in one fundamental polarization at the input, and soliton locking will no longer occur. Hence the threshold for the fundamental excitation of the soliton should diverge when we approach the $e$ - or $o$-excitation polarization input, the onset of which can be seen in Fig. 2.

Because of the method of excitation, the composition of the QSS's can be expected to differ from that predicted for the equal-fundamentals existence curve. ${ }^{3,13}$ In Fig. 3 the fractions of the energy that appear at the exit face of the crystal as a trapped $o$-polarized fundamental, an $e$-polarized fundamental, and a second harmonic are shown for a phase mismatch of 0 . The generated soliton has marginally more energy in the two fundamental components than in the harmonic. Again we note that the generated solitons are not on the minimum existence curve defined by Torner et $a l .{ }^{13}$ The variation in the soliton composition with input polarization shows that the family of QSS's generated in this way is dramatically different from the balanced fundamental excitation case. Specifically, solitons could be generated with one dominant fundamental component that contains $>70 \%$ of the total soliton energy. At the different phase mismatches studied the harmonic contribution was al- ways the greatest with balanced fundamental inputs. For increasing values of imbalance, the fraction of the second harmonic decreased monotonically. Furthermore, the largest component of the soliton that was generated corresponds to the largest component at the input. Therefore it appears possible to generate QSS's with large ratios of the two fundamental components. Thus, for example, one could consider a signal beam guided by a control beam.

All of these QSS composition experiments were performed at a constant input energy above the minimum threshold for all imbalances studied. We performed an additional experiment to study the dependence of the soliton composition with increasing input energy at a fixed phase mismatch and polarization angle. No variation in the soliton composition was observed to within the experimental uncertainty.

Figure 4 illustrates the compositional variety of this QSS family. The triangular axes of Fig. 4 are particularly well suited to represent the three-coupledwave structure. Each extremum of the diagram represents one component: the bottom left is the fundamental $o$-polarized field, the bottom right is the fundamental $e$-polarized field, and the top is the second-harmonic field. A point inside the triangle identifies a given composition, and the fraction in each wave is obtained by projection of the point upon the three outside axis (following the axes as indicated in the figure). Solitons generated through initial



Fig. 2. Soliton-formation threshold energy ( $\mathbf{\square})$ for linearly polarized fundamental-frequency inputs with various imbalances between extraordinary and ordinary fundamental energy at zero phase mismatch. Also shown is the composition of the fundamental pulse energy in terms of the $e(\triangle)$ and $o(\nabla)$ polarization.

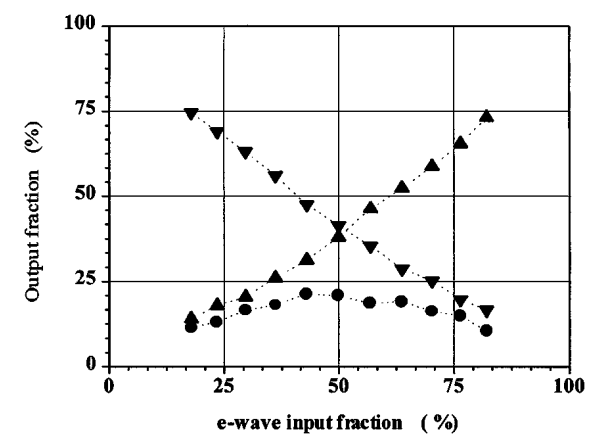

Fig. 3. Output composition of QSS: fraction of harmonic $(\boldsymbol{O})$ and fundamental $e(\mathbf{\Delta})$ and $o(\boldsymbol{\nabla})$ waves as a function of the fraction of the input fundamental in the $e$ polarization for a phase mismatch of zero (total fundamental input, $13 \mu \mathrm{J})$. 


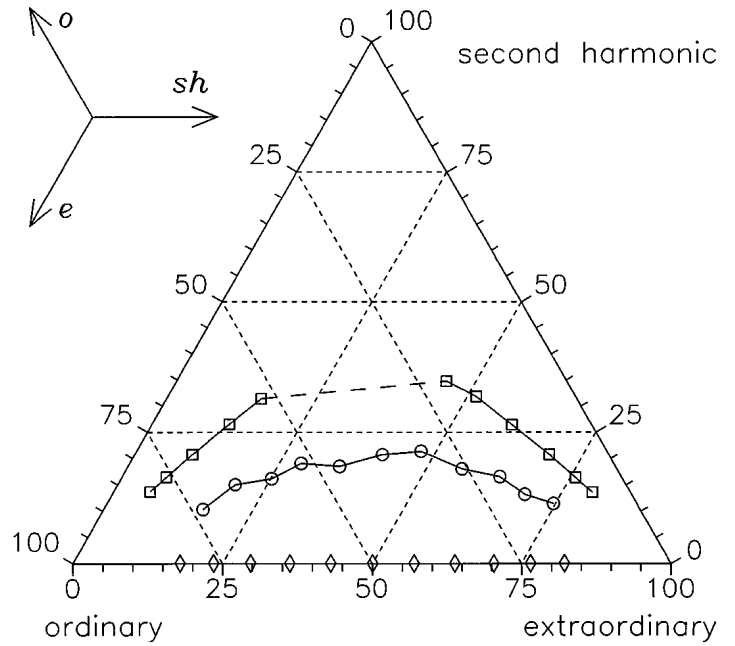

Fig. 4. Structural ternary-composition diagram for quadratic solitons, as explained in the text. Output experimental $(\mathrm{O}$; pulsed, $13 \mu \mathrm{J})$ and corresponding calculated (cw) compositions ( $\square$ ) are shown for a phase mismatch of zero. The corresponding composition of the fundamentals that are launched is indicated for each data point along the bottom axis $(\diamond)$.

upconversion of balanced fundamental input ${ }^{4}$ or downconversion of the second harmonic ${ }^{6}$ could be plotted on the middle vertical axis, representing the family with different amounts of second-harmonic power but equal fundamental $e$ - and $o$-polarized powers. The experimental data for imbalanced fundamental inputs with a total energy of $13 \mu \mathrm{J}$ and a zero phase mismatch are plotted with open circles in Fig. 4. The deviation from the central axis of this plot illustrates the diverse QSS family that can be generated.

This type II interaction between a second harmonic and the fundamentals can be modeled by the usual coupled mode equations for three-wave mixing with two of the waves degenerate in frequency but not polarization in the $\mathrm{cw}$ approximation. ${ }^{4}$ Numerical simulations of the coupled mode equations were performed with a split-step beam-propagation method. We used a $256 \times 256$ grid with 1000 propagation steps for five diffraction lengths. The boundaries were set $\sim 10$ beam widths from the center, and we used an absorbing filter to minimize their influence.

The experimental observations (for pulsed inputs) in Fig. 4 were in good qualitative agreement with the $\mathrm{cw}$ numerical simulations. Also plotted in Fig. 4 on the fundamental (bottom) axis is the initial launching composition. The trapping process is fairly efficient, and because equal amounts of photons were taken from each polarization to form the second-harmonic wave, the output fundamental imbalance becomes slightly larger than that at the input. Thus formation of QSS's follows a fanlike evolution line, and their composition is stabilized on concave curves that are specific for a given detuning and QSS power.

Also, the numerical simulations revealed that if we gradually change the input composition of the beam, we obtain a jump in the composition of the output soliton. This jump occurs for an initial imbalance that corresponds to an approximate $e$-beam-o-beam- fundamental ratio of $49 / 51$ and is responsible for the output position jump reported in Ref. 7 . These previous experiments were done for a small range of imbalance and near-threshold conditions. The current experiments were performed at constant power over a wide range of imbalance, and were thus well above threshold around the balanced conditions, which lead to a large amount of background radiation, masking the jump in composition for the experimental data.

In summary, the properties of type II QSS's have been investigated by excitation of only the two fundamental waves for a wide range of input-energy imbalance between the two polarizations. An extensive family of QSS's was found with a continuous ratio between the energies of the two orthogonally polarized fundamental waves. The fundamental pulse threshold energy for the excitation of this branch is minimum for equal fundamental beam excitation and increases with a decreasing fraction of any of the fundamental beams. Numerical simulations support the experimental results. Such type II QSS's could be used for trapping of the fundamental wavelength of a signal beam with a control beam, with only small losses owing to conversion of the fundamental to the second harmonic, for optical information processing.

This research was supported by the National Science Foundation, the Defense Advanced Research Projects Agency, the U.S. Army Research Office, and the U.S. Air Force Office of Scientific Research. M. Canva acknowledges support from the French Direction Generale de l'Armament under contract ERE 96-1101.

\section{References}

1. Y. Karamzin and A. Sukhorukov, Zh. Eksp. Teor. Phys. 68, 834 (1975) [Sov. Phys. JETP 41, 414 (1976)].

2. D. Pelinovsky, A. Buryak, and Y. Kivshar, Phys. Rev. Lett. 75, 591 (1995).

3. L. Torner, C. Menyuk, W. Torruellas, and G. Stegeman, Opt. Lett. 20, 13 (1995).

4. W. Torruellas, Z. Wang, D. Hagan, E. Van Stryland, G. Stegeman, L. Torner, and C. Menyuk, Phys. Rev. Lett. 74, 5036 (1995).

5. R. Schiek, Y. Baek, and G. Stegeman, Phys. Rev. E 53, 1138 (1996).

6. M. Canva, R. Fuerst, D. Baboiu, G. Stegeman, and G. Assanto, Opt. Lett. 22, 1683 (1997).

7. W. Torruellas, G. Assanto, B. Lawrence, R. Fuerst, and G. Stegeman, Appl. Phys. Lett. 68, 1449 (1996).

8. Y. Baek, R. Schiek, G. Stegeman, I. Baumann, and W. Sohler, Opt. Lett. 22, 1550 (1997).

9. U. Peschel, C. Etrich, F. Lederer, and B. Malomed, Phys. Rev. E 55, 7704 (1997).

10. A. V. Buryak, Y. S. Kivshar, and S. Trillo, Phys. Rev. 77, 5210 (1996).

11. R. Mollame, S. Trillo, and G. Assanto, Opt. Lett. 21, 1969 (1996); A. D. Capobianco, B. Costantini, C. De Angelis, A. Laureti-Palma, and G. F. Nalesso, IEEE Photon. Technol. Lett. 9, 602 (1997).

12. R. Fuerst, B. Lawrence, W. Torruellas, and G. Stegeman, Opt. Lett. 22, 19 (1997).

13. L. Torner, D. Mihalache, D. Mazilu, E. Wright, W. Torruellas, and G. Stegeman, Opt. Commun. 121, 149 (1995). 